\title{
Molecular classification reveals the diverse genetic features and prognosis of gastric cancer: a multi-omics consensus ensemble clustering
}

1 Xianyu Hu ${ }^{1 \#}$, Zhenglin Wang ${ }^{1 \#}$, Qing Wang ${ }^{2 \#}$, Ke Chen ${ }^{1}$, Qijun Han ${ }^{1}$, Suwen Bai ${ }^{3}$,

2 Juan $\mathrm{Du}^{3,4^{*}}$, Wei Chen ${ }^{1^{*}}$

31 Department of General Surgery, The First Affiliated Hospital of Anhui Medical

4 University Hefei, Anhui Province 230022, P.R. China

52 Department of Biliary-Pancreatic Minimally Invasive Surgery, The First Affiliated

6 Hospital of Shantou University Medical College, Shantou, Guangdong Province 515000,

$7 \quad$ P.R. China

83 Longgang District People's Hospital of Shenzhen \& The Third Affiliated Hospital

9 (Provisional) of The Chinese University of Hong Kong, Shenzhen, Shenzhen,

10 Guangdong Province 518172, P.R. China

114 School of Medicine, The Chinese University of Hong Kong, Shenzhen, Shenzhen,

12 Guangdong Province 518172, P.R. China

14 \# These authors contributed equally to the study.

15 Correspondence should be addressed to Wei Chen (chenwei366@ahmu.edu.cn) 
$19{ }^{*}$ Correspondence to:

20 Prof. Juan Du

21 Longgang District People's Hospital of Shenzhen \& The Third Affiliated Hospital

22 (Provisional) of The Chinese University of Hong Kong, Shenzhen, Shenzhen,

23 Guangdong Province 518172, P.R. China

24 School of Medicine, The Chinese University of Hong Kong, Shenzhen, Shenzhen,

25 Guangdong Province 518172, P.R. China

26 Longxiang Road 2001, Shenzhen, Guangdong Province, P.R. China

27 Tel./Fax: +86-755-23516109

28 dujuan@cuhk.edu.cn

29 Prof. Wei Chen

30 Department of General Surgery, The First Affiliated Hospital of Anhui Medical

31 University Hefei, Anhui Province 230022, China

32 Jixi Road 218, Hefei, Anhui Province 230022, P.R. China

33 Tel./Fax: +86-551-62922813

34 chenwei366@ahmu.edu.cn

35 Running title: Novel molecular classification of gastric cancer 


\section{Abstract:}

38 Background: Gastric cancer (GC) is the fifth most common tumor around the

39 world, it is necessary to reveal novel molecular subtypes to guide the selection of

40 patients who may benefit from specific target therapy.

41 Methods: Multi-omics data, including RNA-sequence of transcriptomics (mRNA,

42 LncRNA, miRNA), DNA methylation and gene mutation of TCGA-STAD cohort

43 was used for the clustering. Ten classical clustering algorithms were applied to 44 recognize patients with different molecular features via the R package "MOVICS". The

45 activated signaling pathways were evaluated using the single-sample gene set 46 enrichment analysis. The difference distribution of gene mutations, copy number

47 alterations and tumor mutation burden was compared, and potential response to 48 immunotherapy and chemotherapy was assessed as well.

49 Results: Two molecular subtypes (CS1 and CS2) were recognized by ten clustering

50 algorithms with further consensus ensembles. Patients in the CS1 group were found to 51 contain a shorter average overall survival time (28.5 vs. 68.9 months, $P=0.016$ ), and 52 progression-free survival (19.0 vs. 63.9 months, $P=0.008)$ compared to the CS2 group.

53 CS1 group contained more activation of extracellular associated biological process, 54 while CS2 group displayed the activation of cell cycle associated pathways. The 55 significantly higher total mutation numbers and neo antigens were observed in CS2 56 group, along with the specific mutation of TTN, MUC16 and ARID1A. Higher infiltration

57 of immunocytes were also observed in CS2 group, reflected to the potential benefit from 58 immunotherapy. Moreover, CS2 group also can response to 5-fluorouracil, cisplatin, and 
59 paclitaxel. The similar diverse of clinical outcome of CS1 and CS2 groups were

60 successfully validation in external cohorts of GSE62254, GSE26253, GSE15459, and

61 GSE84437.

62 Conclusion: Novel insight into the GC subtypes was obtained via integrative analysis of

63 five omics data by ten clustering algorithms, which can provide the idea to the clinical

64 target therapy based on the specific molecular features.

65 Keywords: gastric cancer, molecular classification, multi-omics, overall survival, 66 gene mutation

\section{Introduction}

68 Gastric cancer (GC) is the fifth most common tumor and ranks third in mortality

69 worldwide[1]. China is the most affected country by GC, accounting for $42.6 \%$ of global

70 incidence and $45 \%$ of GC-related death. In addition, it is second in terms of incidence

71 among all malignancies in China[2, 3]. Helicobacter pylori infection is the most important

72 pathogenic factor of GC. Most patients infected with Helicobacter pylori remain

73 asymptomatic, however, almost all patients' symptoms are accompanied with varying

74 degrees of atrophic gastritis and intestinal metaplasia[4]. For most early GC patients,

75 endoscopic resection is a definitive treatment[5]. For non-early GC patients, gastrectomy

76 with D2 lymphadenectomy and chemotherapy is an internationally agreed upon

77 treatment[6-8]. Recently, combination adjuvant chemotherapy in both the preoperative

78 and postoperative period have been considered in being beneficial for GC patients[9, 10].

79 The Cancer Genome Atlas (TCGA) group has divided four molecular subtypes of GC,

80 including Epstein-Barr virus (EBV)-positive, microsatellite instability (MSI), genomically 
81 stable (GS) and chromosomal instability (CIN)[11]. The EBV-positive subtype has

82 demonstrated enrichment of 9p amplification with consecutive high expression of PD-L1

83 and PD-L2. PD-1/PD-L1 inhibitors, which may have alleviating effects in EBV-positive

$84 \mathrm{GC}[12]$. The MSI subtype is characterized by mutations in the DNA mismatch repair

85 system (MMR) or silencing of respective promoter regions by hypermethylation, which

86 can detect and repair DNA base pair mismatches. In patients with resectable primary

$87 \mathrm{GC}, \mathrm{MSI}$ serves as a robust biomarker that indicates favourable post-surgical survival

88 outcomes[13]. GS is generally a diffuse GC, in which $\mathrm{CDH} 1$ and RHOA mutations are

89 detectable or frequently show CLDN18-ARHGAP fusion[14]. Moreover, TP53 mutations

90 are found in $70 \%$ of CIN GCs, which may result in aneuploidy and focused amplification

91 of receptor tyrosine kinases[15]. The Asian Cancer Research Group (ACRG) has

92 reported four expression subtypes of GC: MSS/TP53-, MSS/TP53+, MSI, and

93 MSS/EMT, in which MSS refers to microsatellite stable tumors. Among these molecular

94 subtypes, MSI has the best prognosis, while MSS/EMT has the poorest prognosis and

95 MSS/TP53- and MSS/TP53+ have intermediate prognosis[16].

96 Currently, only two biomarkers are available to predict the therapeutic effect of patients:

97 human epidermal growth factor receptor 2 (HER2) for trastuzumab and programmed

98 death-ligand 1 (PD-L1) expression for pembrolizumab[17-19]. Although several

99 immunotherapy-based trials have reported widespread tumor response rates in GC

100 patients, many phase III trials of targeted drugs have not shown significant survival

101 benefits[20, 21]. Weak selection of patient molecules included in clinical trials remains

102 an issue and may limit the evaluation of the benefits of many therapeutic drugs, such as

103 anti-angiogenic molecules and newer immunomodulatory agents. Therefore, it is

104 necessary to uncover novel biomarkers in order to better select patients who may benefit

105 from specific target therapy. 


\section{Preparing the multi-omics data of the TCGA-STAD cohort}

The TCGA-STAD cohort was chosen as the discovery cohort as it has multi-omics data,

109 such as the bulk RNA-sequence of transcriptomics (mRNA, LncRNA, miRNA), DNA

110 methylation and gene mutation. In addition, the expression data of transcriptomics were

111 downloaded using the R package "TCGAbiolinks", while the gene symbols of mRNA

112 were annotated using the GENCODE27 annotation file and the LncRNAs were identified

113 by Vega. The expression of miRNAs was downloaded from UCSC Xena (), in which the

114 mature miRNA names were renamed using the R package "miRNAmeConverter" along

115 with the miRbase 21.0. The counts data of transcriptomics were then transferred to the

116 transcripts per kilobase million (TPM) value for subsequent analysis. Additionally,

117 Illumina DNA methylation 450 data was downloaded from UCSC Xena, while somatic

118 mutations were obtained from the cBioPortal (). The clinicopathological information of

119 the TCGA-STAD cohort was also obtained using "TCGAbiolinks". A total of 243 GC

120 patients were finally enrolled, who all possessed the types of omics data.

\section{Preparing the external validation cohort}

122 In order to verify the new finding from the discovery cohort, the mRNA expression matrix

123 and clinical information were also downloaded from four external GEO cohorts, including

124 GSE62254, GSE26253, GSE15459 and GSE84437. The GSE62254 cohort contained

125 the microarray profiles from 300 histologically confirmed gastric adenocarcinoma

126 patients from the ACRG gastric cohort. Meanwhile, the GSE26253 cohort contained a

127 microarray gene expression profile of archived paraffin-embedded tumor blocks from

128432 gastric adenocarcinoma patients. GSE15459 recorded a genome-wide mRNA 
129 expression profile of 192 primary gastric tumors from Singapore patients. Moreover,

130 GSE84437 contained the data of 433 gastric tumor patients from Yonsei University. The

131 microarray platform of GSE62254 and GSE26253 was obtained from Affymetrix Human

132 Genome U133 Plus 2.0 Array, Illumina HumanRef-8 WG-DASL v3.0 for GSE15459, and

133 Illumina HumanHT-12 V3.0 expression beadchip for GSE84437.

\section{Identifying molecular subtypes via integrative analysis}

135 In conjunction with guidelines from the recently published R package "MOVICS"[22], the

136 molecular subtypes were identified according to the above-mentioned multi-omics data.

137 The OS related factors were first evaluated by univariate Cox regression, including 138 mRNA, LncRNA, miRNA and DNA methylation CpG sites. In regard to the mutant genes, 139 genes with a mutant frequency higher than $10 \%$ were selected. In order to find the 140 appropriate number of subtypes, analysis of clustering prediction index (CPI) [23] and 141 Gaps-statistics[24] based on the multi-omics data were performed. Subsequently, ten 142 clustering algorithms were used to separate the patients according to different subtypes,

143 after which the combined classification was finally obtained in view of the concept of 144 consensus ensembles to identify the subtypes with high robustness. The ten clustering 145 algorithms contained iClusterBayes, moCluster, CIMLR, IntNMF, ConsensusClustering, 146 COCA, NEMO, PINSPlus, SNF, and LRA. Quantification of sample similarity in the 147 subtypes was also assessed using the silhoutte score.

\section{Evaluating the activation of signaling pathways and immune infiltration}

149 The subtype-specific upregulated and downregulated genes were calculated using the R 150 package "limma", with significant threshold in both the nominal and adjusted P value < 151 0.05. The activation of Gene Ontology (GO) terms of each patient were then evaluated 
152 using the single-sample gene set enrichment analysis (ssGSEA) by the R package

153 "GSVA". The primary result of the gene set enrichment analysis is the enrichment score

154 (ES), which reflects the degree to which a gene set is overrepresented at the top or

155 bottom of a ranked list of genes. A positive ES indicates gene set enrichment at the top

156 of the ranked list, while a negative ES indicates gene set enrichment at the bottom of the

157 ranked list. The normalized enrichment score (NES) is the primary statistic for examining

158 gene set enrichment results. The false discovery rate (FDR) is the estimated probability

159 that a gene set with a given NES represents a false positive finding. The nominal $p$ value

160 estimates the statistical significance of the enrichment score for a single gene set. The

161 gene sets of the GO biological processes were downloaded from the Molecular

162 Signatures Database (MSigDB, https://www.gsea-msigdb.org/gsea/msigdb/index.jsp).

163 The specific pathways of one subtype should be significantly different from the other

164 subtypes. The mean of the enrichment score for each subtype was used in the

165 visualization of the heatmap. The gene sets of immune signatures and stromal 166 signatures were obtained from a previous study[25], and the NES score was calculated 167 in order to compare the different immune activated statuses among the different 168 subtypes.

\section{Evaluating the response to immunotherapy and chemotherapy}

170 In order to evaluate the individual likelihood of responding to immunotherapy, the Tumor 171 Immune Dysfunction and Exclusion (TIDE) algorithm was employed. Moreover, 172 according to a melanoma cohort who received anti-CTLA-4 or anti-PD-1 checkpoint 173 inhibition therapy, specific gene sets were obtained with 795 genes[26]. Subclass 174 mapping analysis was then conducted in order to compare the similarity of risk groups 175 with the immunotherapy subgroups and point out the responders of anti-CTLA-4 or anti- 
176 PD-1 immunotherapy[27]. The chemotherapeutic response for each sample was then

177 predicted based on the Genomics of Drug Sensitivity in Cancer (GDSC). Accordingly, 3

178 commonly used chemo drugs, 5-Fluorouracil, cisplatin, and paclitaxel were selected.

179 The response to the above six drugs was compared by estimating the samples' half-

180 maximal inhibitory concentration $\left(\mathrm{IC}_{50}\right)$ via ridge regression.

\section{Characteristics of genetic alterations among subtypes}

182 The number of nonsynonymous mutations per million bases was then calculated to 183 evaluate the tumor mutation burden (TMB), while FGA referred to the percentage of 184 genome affected by copy number gains or losses. The total mutation numbers and neo 185 antigens were obtained from a previous study[28]. Oncoprint mutation landscape was 186 generated using the R package "maftools" [29]. The cumulative recurrent events were 187 then displayed using the R package "Survival". The copy number segment data were 188 downloaded from FireBrowse (http://firebrowse.org/) and displayed by the R package 189 "maftools".

\section{Statistical analysis}

191 All analyses were completed using the R software v4.0.3 (http://www.r-project.org). 192 Comparisons of continuous data between two groups were performed using the 193 Student's T-test or Wilcoxon test. Pearson correlation coefficient test was then employed 194 in order to assess the relationship between the two factors. The distribution of 195 categorical variables between groups were then compared using the Chi-square test. K-

196 M survival analysis was performed to explore the survival difference between high-risk 197 score group and low-risk score. A log-rank test was used to estimate the survival 198 analysis. $\mathrm{HR}$ and $95 \% \mathrm{Cl}$ were calculated by the cox model. Meanwhile, the independent 
199 prognostic effect of risk score was calculated by multivariate cox regression analysis. P

$200<0.05$ was considered to be a statistically significant difference.

\section{Results}

\section{GC patients were separated to two subtypes by MOVICS}

203 In view of the results of CPI and Gaps-statistics analysis, the highest average value of

204 these two methods when the number of subtypes was found to be 2 (Figure 1A).

205 Subsequently, the integration of the clustering results derived from the 10 algorithms

206 was performed via consensus ensembles. To make the classification more robust

207 (Figure 1B), patients were divided into cluster 1(CS1) and cluster 2 (CS2). Moreover, the

208 similarity of the samples in the identified clusters was also evaluated by silhouette

209 analysis, in which the silhouette score of CS1 was found to be 0.55 while that of CS2

210 was 0.61 . Accordingly, the results demonstrated that the clusters were well apart from

211 each other and were clearly distinguished (Figure 1C). In regard to the distribution of the

212 multi-omics data for the classification, the different distribution of mRNA, LncRNA,

213 miRNA, DNA methylation CpG sites and mutant genes were visualized, as shown in

214 Figure 1D, of which the top 10 OS-associated factors of each omics are displayed in the

215 right, while the clinical features of grade, stage, age and gender are also listed.

216 CS1 patients suffered from shorter overall survival time and progression-free

217 survival

218 The clinical prognosis outcome of GC patients in CS1 and CS2 were further compared.

219 Accordingly, patients in the CS1 group were found to contain a shorter average OS time

220 compared to the CS2 group (28.5 vs. 68.9 months, $P=0.016$, Figure $2 \mathrm{~A}$ ). A similar

221 difference in PFS time was also observed, in which the average PFS time of the CS1 
222 group was significantly shorter than that of the CS2 group (19.0 vs. 63.9 months, $\mathrm{P}=$

2230.008 , Figure 2B). The distribution of different clinicopathological features in CS1 and

224 CS2 groups were also compared (Table 1). Patients in the CS1 group contained more 225 white patients $(P=0.007)$, more patients live with tumors $(P=0.045)$, and low average 226 age $(P=0.027)$, while the distribution of tumor stage, grade and gender showed no 227 differences (all $P>0.05$ ). The distribution of different factors in separated subtypes are 228 given in Figure 2C.

Different expressed genes and signaling pathways in CS1 and CS2

230 To characterize the features of CS1 and CS2, the different expressed genes (DEGs) 231 between the CS1 and CS2 groups were first identified. Based on the "limma" package, 2322016 upregulated genes were selected from the CS1 group, while 400 upregulated 233 genes were taken from the CS2 group with a threshold $\mathrm{P}$ value $<0.05$ and fold-change $>$

2342 (Table S). With the help of Metascape, the DEGs were annotated and enriched to 235 biological processes. For CS1 activated pathways, the activation of extracellular 236 associated biological process were mostly observed, including epithelial mesenchymal 237 transition, cell junction organization, cellular component morphogenesis, response to 238 growth factor and cell-substrate adhesion pathways (Figure 3A). In regard to the CS2 239 group, pathways of cell cycle, including G2M checkpoint, cell cycle, E2F targets, G1/S240 specific transcription, DNA replication and repair biologic process were mainly focused 241 on (Figure 3B).

242 For all of the DEGs among CS1 and CS2, this study focused on SMOC2 (Figure 4A).

243 SMOC2 was found to be significantly increased in the CS1 subtype, with a fold-change 244 of 4.537 (adjusted $P$ value $<0.001$ ). The different activation of extracellular matrix 245 associated pathways were also assessed by GSEA analysis, in which three pathways 
246 were found to be significantly activated in the CS1 subtype compared to the CS2

247 subtype (all $\mathrm{P}<0.01$, Figure 4B). Several studies have reported the function of SMOC2

248 in tumorigenesis through extracellular matrix associated pathways, however, few were

249 concerned with GC patients. Accordingly, a high expression of SMOC2 was observed to

250 be linked with poor prognosis in $\mathrm{GC}$ patients $(\mathrm{HR}=1.66,95 \% \mathrm{Cl}=1.081-2.551, \mathrm{P}=$

251 0.021, Figure 4C). Moreover, the mRNA expression of SMOC2 was found to be

252 positively associated with mesenchymal maker, Vimentin $(R=-0.32, P<0.001$, Figure

253 4D), which was negatively associated with epithelia marker, E-Cadherin $(R=-0.27, P<$

2540.001 , Figure 4D). In addition, the prognostic value of SMOC2 in three GEO cohorts

255 were also evaluated, in which high levels of mRNA of SMOC2 was found to be

256 associated with poor prognosis (GSE15459, $\mathrm{HR}=1.56,95 \% \mathrm{Cl}=1.05-2.32, \mathrm{P}=0.027$;

257 GSE62254, $\mathrm{HR}=2.02,95 \% \mathrm{Cl}=1.40-2.91, \mathrm{P}<0.001 ; \mathrm{GSE} 51105, \mathrm{HR}=1.52,95 \% \mathrm{Cl}$

$258=0.91-2.54, P=0.11 ;$ Figure 4E).

259 CS2 contained more genetic alterations

260 The gene mutation and copy number alteration play pivotal role in the initial stages and 261 development of tumorigenesis. In this regard, the genetic alterations among CS1 and 262 CS2 were then compared.

263 The number of nonsynonymous mutations per million bases was calculated so as to evaluate

264 the TMB. Here, a significantly higher average TMB value was present in CS2 compared to 265 that of CS1 ( $\log 10$ transformed TMB value: 0.68 vs. $0.52, P<0.001$, Figure 5A). Moreover, 266 the total mutation numbers in CS2 were also higher than that of the CS1 group $(P<0.001$, 267 Figure 5B), in conjunction with the numbers of neo antigens ( $P<0.001$, Figure 5C). 268 Additionally, the difference of mutation in a single gene was also compared, where there 269 were more TTN mutations $(65.2 \%$ vs. $42.7 \%, \mathrm{P}<0.001)$, MUC16 mutations $(44.1 \%$ vs. 
$27022.1 \%, P=0.002$ ), and ARID1A mutations (36.6\% vs. 11.5\%, $\mathrm{P}<0.001)$ (Figure 5D, Table

271 2). Mutated MUC16 decreased the cumulative rate of OS events, which acted as a tumour

272 suppressor $(P=0.012$, Figure $5 \mathrm{E})$. Based on the databases from cBioPortal, the total

273 genetic alteration of MUC16 was found to be about $15 \%$ to $30 \%$, in which the altered group

274 demonstrated better prognosis $(P<0.001$, Figure $\mathbf{S 1})$. The copy number alterations were

275 also calculated, in which the CS2 groups were noted to contain more CNAs of both genomes

276 lost or gained (all $\mathrm{P}<0.05$, Figure 5F).

277 CS2 group contained more activated immune infiltration and responded more

\section{8 from immuno-/chemotherapy}

279 Due to the CS2 group contained more gene mutations, CNAs and neo antigens. Hence,

280 it is reasonable to postulate that the CS2 group underwent an activated status of

281 immune infiltration. Based on the calculation of GSEA to immune activation and

282 suppression signatures, the CS2 subtype was found to contain a higher score of

283 activation signatures, including immune enrichment score, cytotoxic cells, activated CD4

284 T cell, activated CD8 T cell, 6 gene IFN signature, and CYT. Meanwhile, an activated

285 status of suppression signatures in the CS1 group was also observed, including MDSC,

286 Macrophages, Wnt/TGF $\beta$ signature, TITR, TGFB1 activated and C-ECM signatures

287 (Figure 6A). Furthermore, the potential response to immunotherapy of these 243 GC

288 patients were evaluated by TIDE, in which a total of 65 patients served as potential

289 responders to immunotherapy (Figure 6B), while more responders belonged to the CS2

290 group ( $32 \%$ vs. $22 \%, P=0.083$, Figure 6 C). However, the potential response to chemo

291 drugs commonly used in GC patients was also assessed, which showed that the CS2

292 group responded more to treatment with 5 -fluorouracil, cisplatin, and paclitaxel (all $\mathrm{P}<$

293 0.01, Figure 6D). 
295 Based on the "limma" package, the top 200 upregulated biomarkers were identified 296 (Figure S2) for CS1 and CS2, respectively, with a significance threshold (adjusted P < 297 0.05) and no overlap with any biomarkers identified for other subtypes. The four external 298 cohorts were noted to be GSE62254, GSE26253, GSE15459, and GSE84437, 299 containing a total of 1357 GC patients. By employing the NTP method, the CS1 and CS2 300 group was predicted for each cohort by subtype-specific upregulated biomarkers, 301 respectively (Figure 7A). Notably, the consistent prognosis results of CS1 and CS2 302 were illustrated in all of the four external GEO cohorts. Patients in the CS1 group had a 303 more unfavorable OS outcome, while those in the CS2 group faced a favorable 304 prognosis (GSE62254, $\mathrm{HR}=0.53,95 \% \mathrm{Cl}=0.38-0.72, \mathrm{P}<0.001 ; \mathrm{GSE} 26253, \mathrm{HR}=$ $3050.71,95 \% \mathrm{Cl}=0.52-0.96, \mathrm{P}=0.027 ; \mathrm{GSE} 15459, \mathrm{HR}=0.61,95 \% \mathrm{Cl}=0.41-0.92, \mathrm{P}=$ 306 0.018; GSE84437, $\mathrm{HR}=0.59,95 \% \mathrm{Cl}=0.45-0.78, \mathrm{P}<0.001$; Figure 7B).

Discussion

308 Several studies have reported the molecular subtypes of GC patients in order to 309 illustrate the tumor heterogenies and guide clinical treatment. The Cancer Genome Atlas 310 Research Network has proposed a molecular classification to divide gastric cancer into 311 four subtypes: Epstein-Barr virus (EBV), microsatellite instability (MSI), genomically 312 stable (GS) and chromosomal instability (CIN)[11]. EBV subtype displays recurrent 313 PIK3CA mutations, extreme DNA hypermethylation, and amplification of JAK2, PD-L1 314 and PD-L2; MSI subtype shows elevated mutation rates; GS subtype is enriched for the 315 diffuse histological variant and mutations of RHOA; and CIN subtype shows marked 316 aneuploidy and focal amplification of receptor tyrosine kinases. Meng et al.[30] pointed 317 out three GC subtypes that had different DNA methylation modification patterns based 
318 on the DNA methylation of 1619 GC patients, for which high DNA methylation score

319 (DMS) was characterized by immune activation status, increased tumor mutation burden, 320 and tumor neoantigens, which had a favorable prognosis. Meanwhile, the activation of 321 the stroma and absence of immune cell infiltration were observed in the low DMS group, 322 with relatively poor survival. Zhou et al. [31] also refined the molecular subtypes of GC 323 patients by epigenetic age acceleration pattern. The definition of age acceleration (AA) is 324 the difference between biological and chronological age. Patients with positive AA 325 demonstrated better prognosis compared to those with negative AA. Furthermore, 326 patients who belonged to the EBV subtype contained the highest average AA compared 327 to MSI, GS and CIN subtypes. However, few studies were concerned with the 328 classification of GC patients with multi-omics data.

329 In the current study, using the newly developed R package "MOVICS", two molecular 330 subtypes, CS1 and CS2, were identified by applying multi-omics data consistent of 331 mRNA, LncRNA, miRNA, DNA methylation and gene mutation. The number of 2 clusters 332 was selected by the prediction of CPI and Gaps-statistics analysis, and the subsequent 333 separated from the total 243 patients were based on the consensus ensembles of 10 334 clustering algorithms. Interestingly, patients in the CS1 group had a poor prognosis in 335 regard to their OS and RFS, while those in CS2 had a longer OS and RFS time. The 336 activated extracellular matrix and epithelial mesenchymal transition pathways were 337 observed in the CS1 group as well. The molecular pathogenesis of GC was also highly 338 associated with E-cadherin, which harbor genetic and epigenetic abnormalities in both 339 germline and sporadic GCs[32]. Xue et al.[33] put forward that the level of ZEB1 340 expression correlates with the degree of differentiation, metastasis to lymph nodes, and 341 the stage of GC. In addition, suppression of ZEB1 may improve the expression of E342 cadherin in GC cells and reduce the expression of vimentin. The correlation between 
343 SMOC2 and diseases has been widely reported. Jang et al.[34] reported that SMOC2 is

344 a tumor suppressor in colorectal cancer and can elevate the expression of SMOC2 and

345 suppress cell migration, colony and sphere formation. However, Lu et al.[35] reported on

346 the suppression function in colon carcinoma invasion and migration of ARNTL2 via

347 decreased SMOC2-EMT activity. In the current study, highly expressed SMOC2 in the

348 poor prognosis CS1 group was also observed, which, on further study, confirmed that

349 increased SMOC2 indicated poor prognosis in both TCGA-STAD and three GEO cohorts.

350 Moreover, the expression of SMOC2 was closely associated with EMT markers.

351 The higher genetic alternations of the CS2 group were also observed more than that of 352 the CS1 group. Specifically, there were more TTN mutations, MUC16 mutations and 353 ARID1A mutations occurring in the CS2 favorable prognosis group. MUC16 serves as a 354 type I transmembrane mucin protein that is comprised of 3 components: a C-terminal 355 domain, a tandem repeat region, and an extracellular $\mathrm{N}$-terminal section. Cancer antigen 356125 (CA-125), which is used to monitor disease progression in ovarian cancer, is part of 357 the tandem repeat domain[36]. MUC16 expression is associated with proliferation and 358 metastatic potential in tumor cells, and MUC16 is known to inhibit the natural killer cell 359 mediated lysis of tumors[37, 38]. The transcriptomic results from Li et al.'s study support 360 the suggestion that MUC16 mutation may predict sensitivity to anti-PD-1 therapy; 361 MUC16 mutant gastric tumors are immunologically "hot"[39]. Zhao et al. described that 362 mutated MUC16 predicted a better prognosis in GC patients and was potentially 363 associated with PI3K/Akt/mTOR signaling and Myc expression. Furthermore, they 364 validated that the knockdown of MUC16 inhibited PI3K/Akt/mTOR signaling and reduced 365 the protein level of Myc. Additionally, Yang et al.[40] reported the prognostic value of the 366 combined mutations of MUC4, MUC16 and TTN, which rose to 0.782 in the TCGA367 STAD cohort and 0.735 in the external validation FUSCC cohort. In the current study, 
more MUC16 mutations for good prognosis of the CS2 subtype were identified, in which mutated MUC16 were found to reduce death events for GC patients. Also, the CS2

370 group with activated infiltration of immunocytes, high expression of PD-1, and high 371 response rate to immunotherapy was revealed.

372 There are several new findings and advantages of note in the current study. First,

373 subtypes with diverse clinical outcome, immune infiltration status, genetic alterations

374 were identified using integrative analysis of multi-omics data. Second, 10 clustering 375 algorithms were enrolled, which further generated integration subtypes via the 376 consensus ensembles. Therefore, the current classification is more robust than other 377 studies, which only used one type of classification method. Third, it was revealed that 378 the CS1 group had a poorer prognosis with less genetic alterations and activation of 379 EMT associated pathways, whereas the CS2 group contained high TMB, more 380 mutations and CNAs, though it had a favorable prognosis and high response rate to 381 immunotherapy. Fourth, the diverse OS outcome between CS1 and CS2 subtypes were 382 successfully validated in a total of 1357 GC patients from four cohorts, demonstrating 383 the universal applicability of the new classification for clinical use.

\section{Conclusion}

385 In this study, novel insight into the GC subtypes was obtained via integrative analysis of 386 five omics data by utilizing ten state-of-the-art multi-omics clustering algorithms. 387 Accordingly, patients in the CS1 group were found to have a poor prognosis and were 388 linked with activation of EMT-associated signaling, while those in the CS2 group had a 389 high mutation rate, high level of infiltration of activated immunocytes, and high response 390 rate to immunotherapy. 


\section{Acknowledgements}

393 This work was supported by the National Natural Science Foundation of China (grant

394 numbers: 81972539); the Key Research and Development Projects of Anhui Province 395 (grant numbers: 201904a07020055).

\section{Author contributions}

397 Conception and Design: Xianyu Hu, Zhenglin Wang, Juan Du and Wei Chen. Collection 398 and Assembly of Data: Qing Wang, Ke Chen, Qijun Han and Suwen Bai. Data Analysis 399 and Interpretation: Xianyu Hu, Zhenglin Wang, Ke Chen and Suwen Bai. Manuscript 400 Writing: Xianyu Hu, Qing Wang, Qijun Han and Wei Chen. Final Approval of Manuscript: 401 All the authors.

402

\section{Conflict of interests}

404 The authors have declared no conflicts of interest.

\section{Availability of data and materials}

407 All data used in this work can be acquired from the GDC portal 408 (https://portal.gdc.cancer.gov/), Gene-Expression Omnibus (GEO;

409 https://www.ncbi.nlm.nih.gov/geo/). 
411 1. Bray F, Ferlay J, Soerjomataram I, Siegel RL, Torre LA, Jemal A: Global cancer statistics 412 2018: GLOBOCAN estimates of incidence and mortality worldwide for 36 cancers in 413185 countries. CA Cancer J Clin 2018, 68(6):394-424.

414 2. Gao K, Wu J: National trend of gastric cancer mortality in China (2003-2015): a 415 population-based study. Cancer Commun (Lond) 2019, 39(1):24.

416 3. Wang FH, Shen L, Li J, Zhou ZW, Liang H, Zhang XT, Tang L, Xin Y, Jin J, Zhang YJ et al: The Chinese Society of Clinical Oncology (CSCO): clinical guidelines for the diagnosis and

4. Hatakeyama M: Malignant Helicobacter pylori-Associated Diseases: Gastric Cancer and MALT Lymphoma. Adv Exp Med Biol 2019, 1149:135-149.

421 5. Ono H, Yao K, Fujishiro M, Oda I, Nimura S, Yahagi N, lishi H, Oka M, Ajioka Y, Ichinose M et al: Guidelines for endoscopic submucosal dissection and endoscopic mucosal resection for early gastric cancer. Dig Endosc 2016, 28(1):3-15.

424 6. Das M: Neoadjuvant chemotherapy: survival benefit in gastric cancer. Lancet Oncol 2017, 18(6):e307.

$4267 . \quad$ Fujita K, Kanda M, Ito S, Mochizuki Y, Teramoto H, Ishigure K, Murai T, Asada T, Ishiyama A, Matsushita $\mathrm{H}$ et al: Association between Lymphovascular Invasion and Recurrence Gastroenterol 2014, 20(38):13667-13680.

432 9. Choi AH, Kim J, Chao J: Perioperative chemotherapy for resectable gastric cancer: 
434 10. Ilson DH: Advances in the treatment of gastric cancer: 2019. Curr Opin Gastroenterol $435 \quad 2019,35(6): 551-554$.

436 11. Cancer Genome Atlas Research N: Comprehensive molecular characterization of gastric adenocarcinoma. Nature 2014, 513(7517):202-209.

438 12. Xing X, Guo J, Ding G, Li B, Dong B, Feng Q, Li S, Zhang J, Ying X, Cheng X et al: Analysis of PD1, PDL1, PDL2 expression and T cells infiltration in 1014 gastric cancer patients. Oncoimmunology 2018, 7(3):e1356144.

441 13. Pietrantonio F, Miceli R, Raimondi A, Kim YW, Kang WK, Langley RE, Choi YY, Kim KM, Nankivell MG, Morano F et al: Individual Patient Data Meta-Analysis of the Value of

14. Usui G, Matsusaka K, Mano Y, Urabe M, Funata S, Fukayama M, Ushiku T, Kaneda A:

DNA Methylation and Genetic Aberrations in Gastric Cancer. Digestion 2021, 102(1):25-32 .

448 15. Derks S, de Klerk LK, Xu X, Fleitas T, Liu KX, Liu Y, Dietlein F, Margolis C, Chiaravalli AM, Da Silva $\mathrm{AC}$ et al: Characterizing diversity in the tumor-immune microenvironment of distinct subclasses of gastroesophageal adenocarcinomas. Ann Oncol 2020, 31(8):1011-

452 16. Cristescu R, Lee J, Nebozhyn M, Kim KM, Ting JC, Wong SS, Liu J, Yue YG, Wang J, Yu K et al: Molecular analysis of gastric cancer identifies subtypes associated with distinct clinical outcomes. Nat Med 2015, 21(5):449-456.

455 17. Fashoyin-Aje L, Donoghue M, Chen H, He K, Veeraraghavan J, Goldberg KB, Keegan P, 
Advanced or Metastatic Gastric or Gastroesophageal Junction Adenocarcinoma

Expressing PD-L1. Oncologist 2019, 24(1):103-109.

459 18. Lordick F, Shitara K, Janjigian YY: New agents on the horizon in gastric cancer. Ann Oncol 2017, 28(8):1767-1775.

461 19. Pereira MA, Ramos M, Dias AR, Faraj SF, Ribeiro RRE, de Castria TB, Zilberstein B, Alves VAF, Ribeiro U, Jr., de Mello ES: Expression Profile of Markers for Targeted Therapy in Gastric Cancer Patients: HER-2, Microsatellite Instability and PD-L1. Mol Diagn Ther 2019, 23(6):761-771.

465 20. Rodriquenz MG, Roviello G, D'Angelo A, Lavacchi D, Roviello F, Polom K: MSI and EBV Positive Gastric Cancer's Subgroups and Their Link With Novel Immunotherapy. J Clin Med 2020, 9(5). Heterogeneity and Enables Therapeutic Screening. Cell Stem Cell 2018, 23(6):882-897 e811.

472 22. Lu X, Meng J, Zhou Y, Jiang L, Yan F: MoVICS: an R package for multi-omics integration and visualization in cancer subtyping. Bioinformatics 2020.

474 23. Chalise P, Fridley BL: Integrative clustering of multi-level 'omic data based on nonnegative matrix factorization algorithm. PloS one 2017, 12(5):e0176278.

476 24. Hastie T, Tibshirani R, Walther G: Estimating the number of data clusters via the Gap statistic. J Roy Stat Soc B 2001, 63:411-423.

478 25. Meng J, Lu X, Zhou Y, Zhang M, Ge Q, Zhou J, Hao Z, Gao S, Yan F, Liang C: Tumor 
481 26. Chen PL, Roh W, Reuben A, Cooper ZA, Spencer CN, Prieto PA, Miller JP, Bassett RL, 482 Gopalakrishnan V, Wani K et al: Analysis of Immune Signatures in Longitudinal Tumor Samples Yields Insight into Biomarkers of Response and Mechanisms of Resistance to Immune Checkpoint Blockade. Cancer discovery 2016, 6(8):827-837.

485 27. Hoshida Y, Brunet JP, Tamayo P, Golub TR, Mesirov JP: Subclass mapping: identifying common subtypes in independent disease data sets. PloS one 2007, 2(11):e1195.

28. Rooney MS, Shukla SA, Wu CJ, Getz G, Hacohen N: Molecular and genetic properties of tumors associated with local immune cytolytic activity. Cell 2015, 160(1-2):48-61.

29. Mayakonda A, Lin DC, Assenov Y, Plass C, Koeffler HP: Maftools: efficient and comprehensive analysis of somatic variants in cancer. Genome Res 2018, 28(11):17471756.

30. Meng Q, Lu YX, Ruan DY, Yu K, Chen YX, Xiao M, Wang Y, Liu ZX, Xu RH, Ju HQ et al: DNA methylation regulator-mediated modification patterns and tumor microenvironment characterization in gastric cancer. Mol Ther Nucleic Acids 2021, 24:695-710.

31. Zhou YJ, Lu XF, Meng JL, Wang QW, Chen JN, Zhang QW, Zheng KI, Rocha CS, Martins CB, Yan FR et al: Specific epigenetic age acceleration patterns among four molecular subtypes of gastric cancer and their prognostic value. Epigenomics 2021, 13(10):767778.

32. Bure IV, Nemtsova MV, Zaletaev DV: Roles of E-cadherin and Noncoding RNAs in the Epithelial-mesenchymal Transition and Progression in Gastric Cancer. Int J Mol Sci 2019, 20(12).

502 33. Xue Y, Zhang L, Zhu Y, Ke X, Wang Q, Min H: Regulation of Proliferation and Epithelialto-Mesenchymal Transition (EMT) of Gastric Cancer by ZEB1 via Modulating Wnt5a 
505 34. Jang BG, Kim HS, Bae JM, Kim WH, Kim HU, Kang GH: SMOC2, an intestinal stem cell

506 marker, is an independent prognostic marker associated with better survival in 507 colorectal cancers. Sci Rep 2020, 10(1):14591.

508 35. Lu M, Huang L, Tang Y, Sun T, Li J, Xiao S, Zheng X, Christopher O, Mao H: ARNTL2 509 knockdown suppressed the invasion and migration of colon carcinoma: decreased SMOC2-EMT expression through inactivation of PI3K/AKT pathway. Am J Trans/ Res

512 36. Felder M, Kapur A, Gonzalez-Bosquet J, Horibata S, Heintz J, Albrecht R, Fass L, Kaur J, Hu K, Shojaei H et al: MUC16 (CA125): tumor biomarker to cancer therapy, a work in

37. Gubbels JA, Felder M, Horibata S, Belisle JA, Kapur A, Holden H, Petrie S, Migneault M, Rancourt C, Connor JP et al: MUC16 provides immune protection by inhibiting synapse

38. Muniyan S, Haridas D,

D, Chugh S, Rachagani S, Lakshmanan I, Gupta S, Seshacharyulu P, Smith LM, Ponnusamy MP, Batra SK: MUC16 contributes to the metastasis of pancreatic ductal adenocarcinoma through focal adhesion mediated signaling mechanism. Genes Cancer 2016, 7(3-4):110-124.

525 40. Zhao H, Zhang L: MUC16 mutation predicts a favorable clinical outcome and correlates decreased Warburg effect in gastric cancer. Biochem Biophys Res Commun 2018, 506(4):780-786. 


\section{$529 \quad$ Figure legends}

\section{$530 \quad$ Figure 1. Identifying the molecular clusters.}

531 A. Prediction of optimal cluster number of multi-omics clusters by cluster prediction index

532 and Gap-statistics; B. Consensus heatmap based on the 10 integrative clustering 533 algorithms to refine the clusters; C. Quantification of sample similarity using silhouette 534 score based on the consensus ensembles result; D. Comprehensive heatmap for the 535 enrolled multi-omics data with the annotation of clinical features.

537 Figure 2. Differential clinical outcome of two groups.

538 A. Kaplan-Meier survival analysis of the overall survival of the two groups; B. Kaplan-

539 Meier survival analysis of the progression free survival of the two groups; C. The 540 distribution of the clusters with different clinical features.

$542 \quad$ Figure 3. Cluster-specific biomarkers and signaling pathways.

543 A. The activated signaling pathways in the CS1 group enriched and annotated by 2016

544 significantly upregulated genes; B. The activated signaling pathways in the CS2 group 545 enriched and annotated by 2016 significantly upregulated genes. 
548 A. Differently expressed genes among CS2 to CS1 groups; B. Activation of extracellular

549 associated signaling pathways; C. High level of SMOC2 indicated poor prognosis in the

550 TCGA-STAD cohort; D. The expression of SMOC2 positively correlated with epithelial

551 mesenchymal transition biomarkers; E. High level of SMOC2 indicated poor prognosis in

552 the GSE15459, GSE62254, GSE51105 cohorts.

553

554 Figure 5. Diversely genetic alterations among two clusters.

555 A. Comparison of TMB and TiTv among two identified groups of gastric cancer; B. The

556 differential number of gene mutations in the CS1 and CS2 groups; C. The differential

557 number of neoantigens in CS1 and CS2 groups; D. The landscape and top different

558 mutant genes in the CS1 and CS2 groups; E. Cumulative events of death in patients

559 with or without MUC16 mutation; F. Bar plot of fraction genome altered among two

560 clusters.

562 Figure 6. Potential treatment targets for gastric patients in two clusters.

563 A. The activation of immune associated signatures in the CS1 and CS2 subtypes; B. The 564 distribution of immunotherapy responders and non-responders predicted by the TIDE 565 method; C. Patients in the CS2 cluster seem to be more suitable for immunotherapy; D. 5665 -Fluorouracial, cisplatin and paclitaxel seem to be more suitable for CS2 patients. 
bioRxiv preprint doi: https://doi.org/10.1101/2021.06.07.447364; this version posted June 7, 2021. The copyright holder for this preprint (which was not certified by peer review) is the author/funder. All rights reserved. No reuse allowed without permission.

569 A. NTP method was applied to predict the CS1 and CS2 group for each cohort by the

570 subtype-specific upregulated biomarkers; B. K-M plot showing the diverse overall

571 survival in the CS1 and CS2 group of four external cohorts.

572 


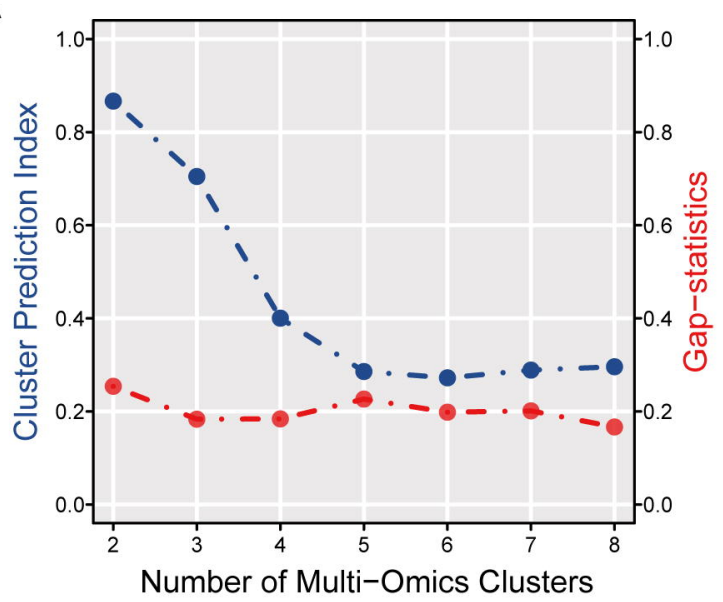

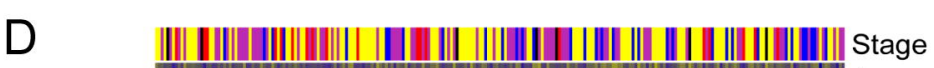
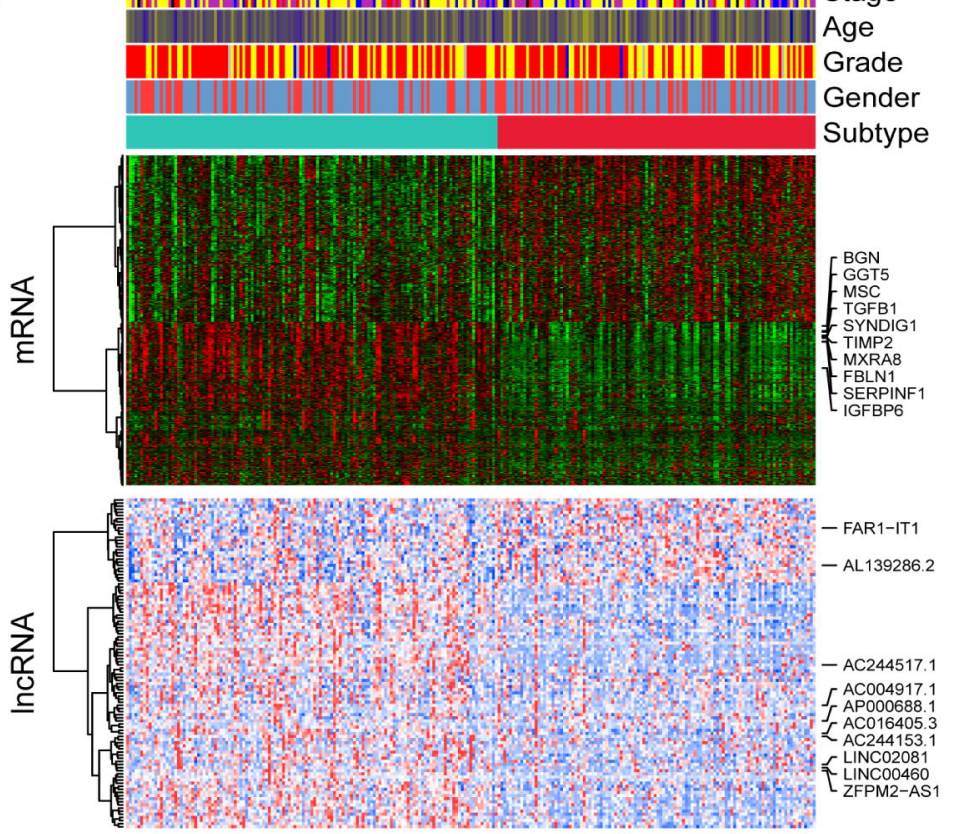

Stage

Stage I

Stage II

Stage III

Stage IV

unknown

Age

Age
$=-100$

$-80$

$-60$

-40
-20

Grade

$\square$ G1

G2

GX
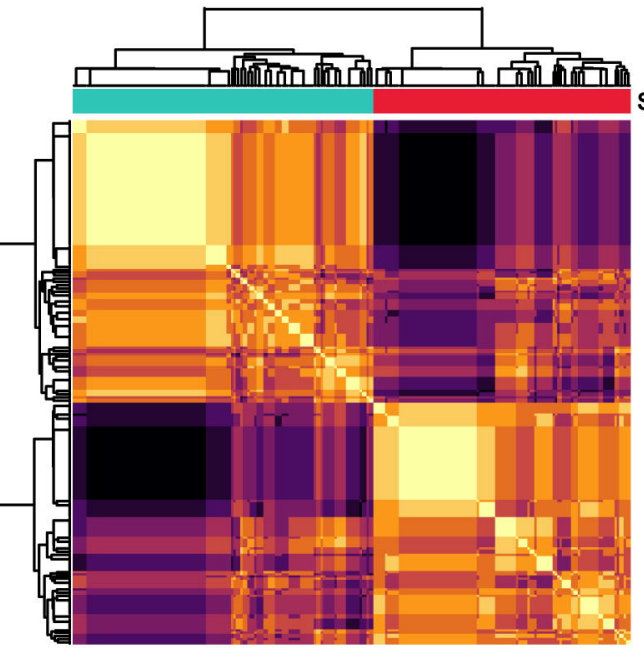

Subtype

C
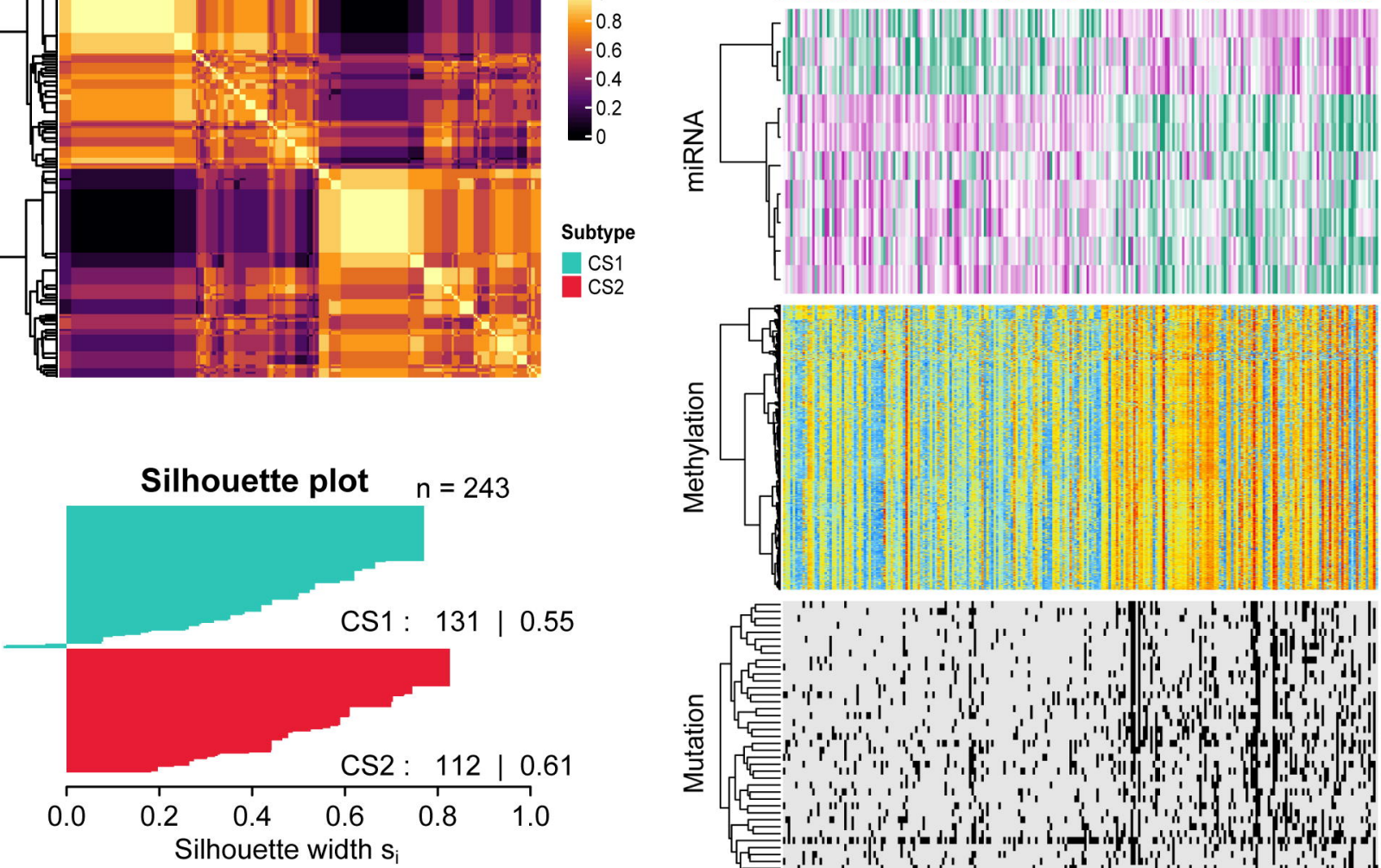

- MIMAT0004489 mRNA.FPKM

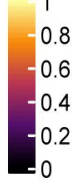

Subtype

CS1

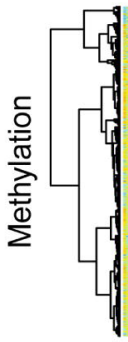

MIMAT0003338

Gender

FEMALE

MALE

Subtype

CS1

- MIMAT0002888

- MIMAT0000098

- MIMAT0000423

- MIMAT0004558

- MIMAT0004814

- MIMAT0000733 $=-2$

- MIMAT0000438

0
-1
--2

miRNA.FPKM

$=2$

$-1$

0

-1
--2

$M$ value

$-2$

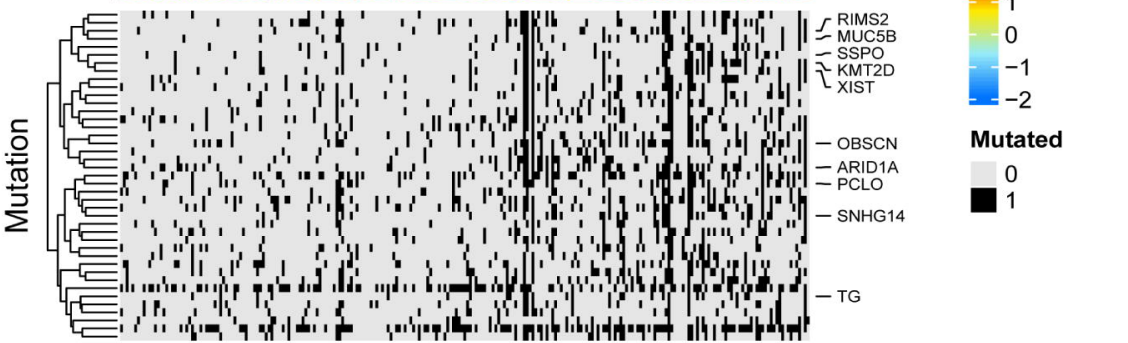

Figure 1 


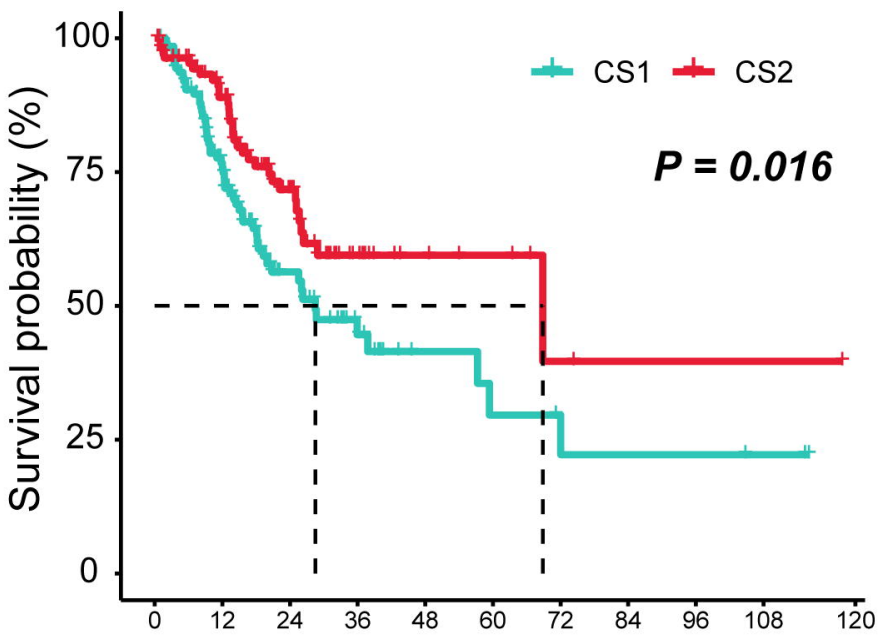

Number at risk

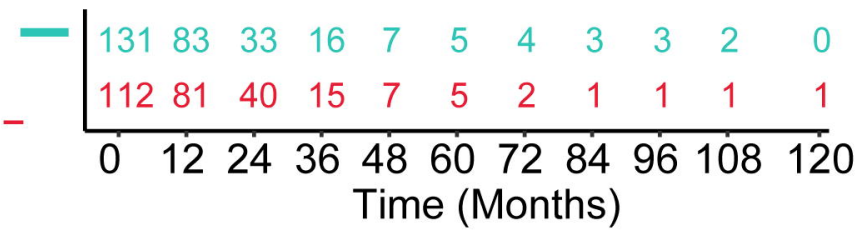

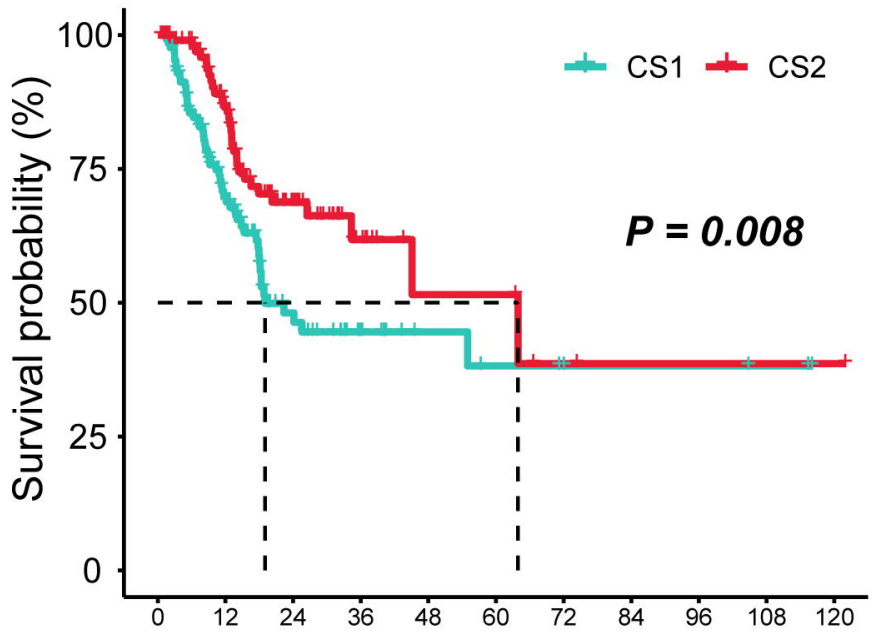

Number at risk

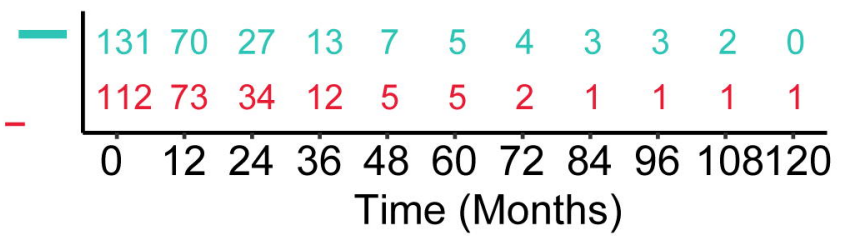

C

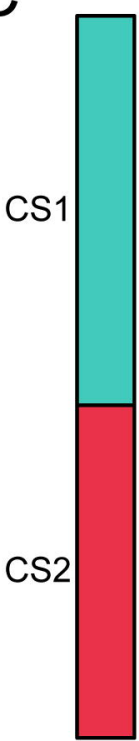

cluster

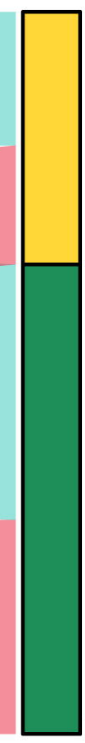

Gender
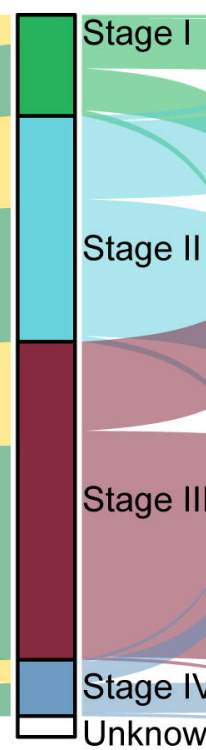

Stage

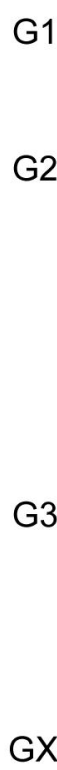

Grade

Figure 2 
A

B

M5901: HALLMARK G2M CHECKPOINT

R-HSA-1640170: Cell Cycle

M5925: HALLMARK E2F TARGETS

GO:0044772: mitotic cell cycle phase transition

GO:0006260: DNA replication

GO:0071103: DNA conformation change

GO:0051321: meiotic cell cycle

GO:0000228: nuclear chromosome

GO:0005813: centrosome

GO:0006281: DNA repair

GO:0008608: attachment of spindle microtubules to kinetochore

GO:0051233: spindle midzone

R-HSA-156711: Polo-like kinase mediated events

R-HSA-69205: G1/S-Specific Transcription

GO:0003682: chromatin binding

R-HSA-174143: APC/C-mediated degradation of cell cycle proteins R-HSA-2514853: Condensation of Prometaphase Chromosomes GO:0045236: CXCR chemokine receptor binding

M5924: HALLMARK MTORC1 SIGNALING

GO:0031023: microtubule organizing center organization
M5930: HALLMARK EPITHELIAL MESENCHYMAL TRANSITION

GO:0001944: vasculature development

GO:0034330: cell junction organization

GO:0003013: circulatory system process

GO:0032989: cellular component morphogenesis

GO:0061061: muscle structure development

M5909: HALLMARK MYOGENESIS

GO:0003012: muscle system process

GO:0070848: response to growth factor

GO:0097435: supramolecular fiber organization

GO:0048729: tissue morphogenesis

I GO:0051272: positive regulation of cellular component movement GO:0001503: ossification

GO:0001501: skeletal system development

GO:0048589: developmental growth

GO:0048598: embryonic morphogenesis

GO:0031589: cell-substrate adhesion

GO:0060485: mesenchyme development

GO:0005539: glycosaminoglycan binding

GO:0005509: calcium ion binding

\section{CS1 activated pathways}

CS2 activated pathways 
A $\quad n=131 \quad n=112$

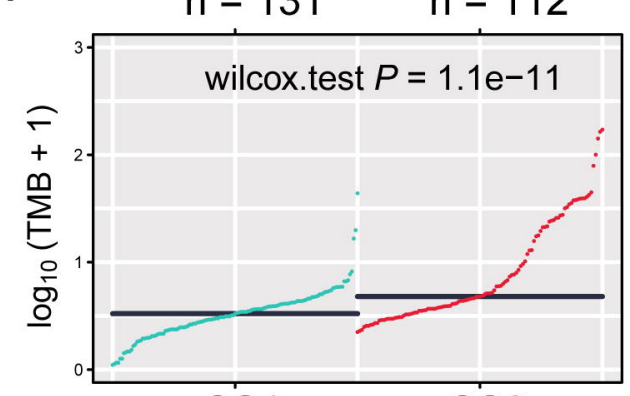

- $C>T$

CS1

$T>C$
$C>A$

- $C>G$

$T>A$

11 $T>G$

D

\section{5}

E

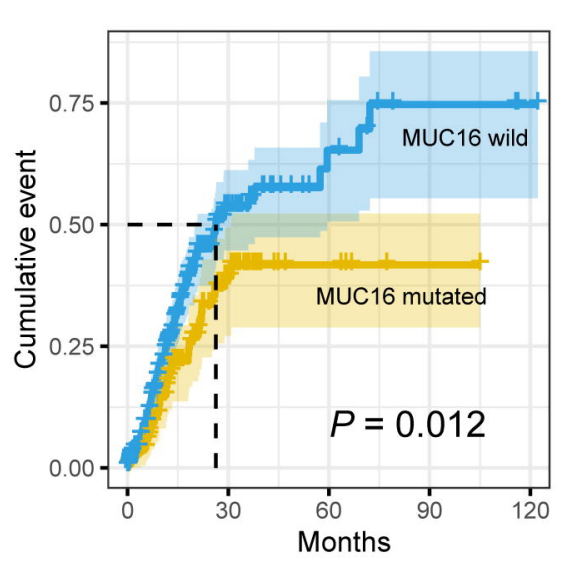

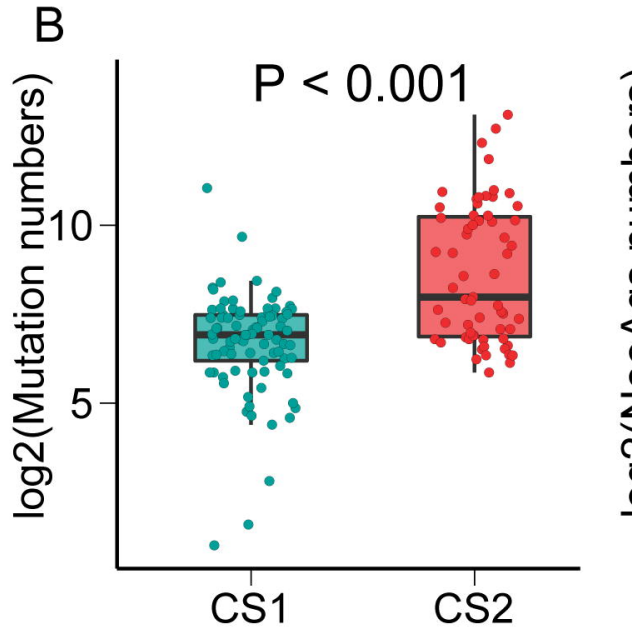

C
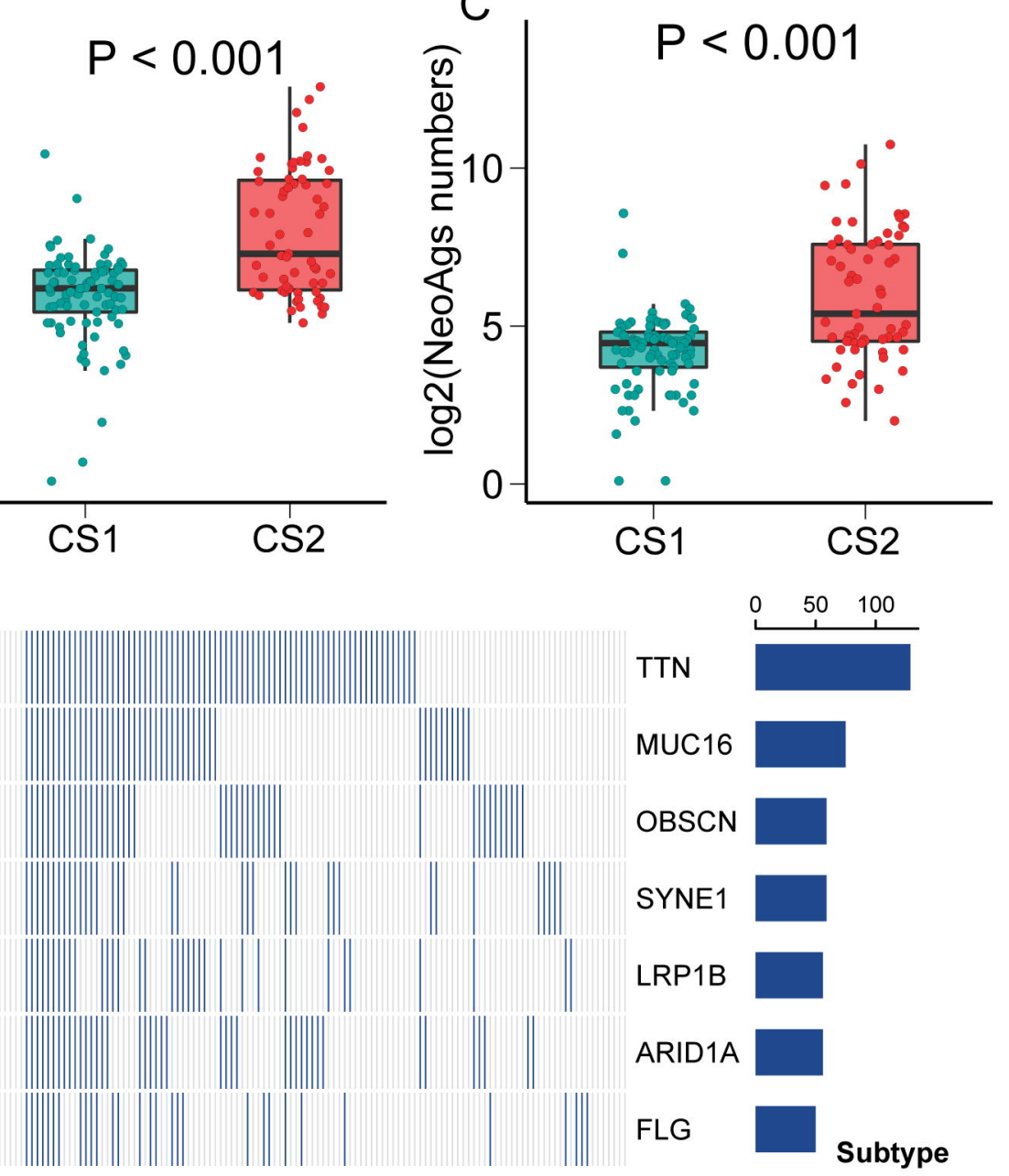

F
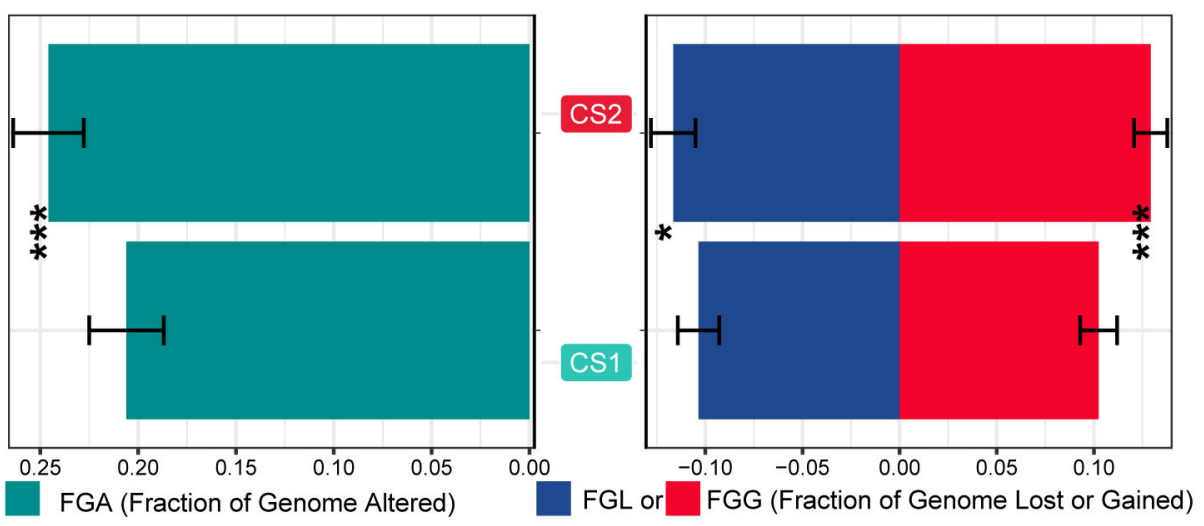

Figure 5 


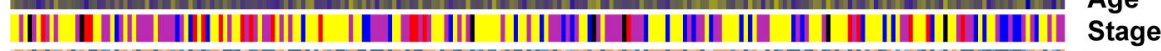

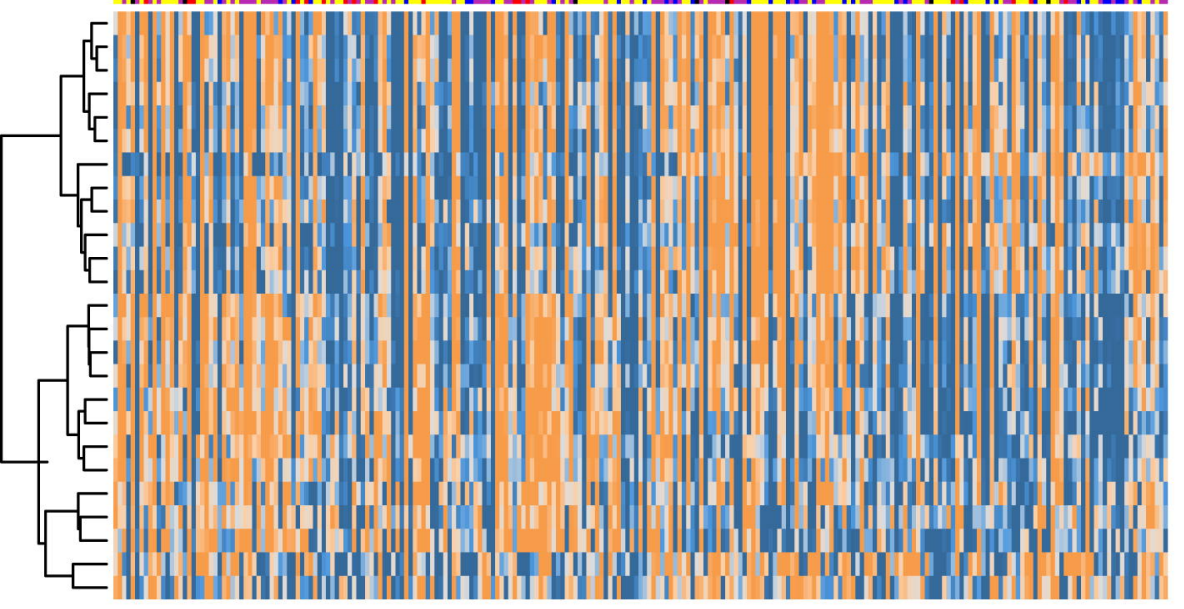

\section{T cells}

Immune enrichment score

T.NK. rileta

Cytotoxic cells

Immune cell subsets

Immune signaling molecules

Activated CD4 T cell

13 T-cell signature

TLS

6 gene IFN signature

Activated CD8 T cell

Type $1 \mathrm{~T}$ helper cell

Regulatory $T$ cell

MDSC

T follicular helper cell

Macrophages

Stromal enrichment score

WNTTGFB signature

AlternativelyactivatedM2

TITR

TGFB1 activated

CECM

PD-1 Signature

Type 17 T helper cell

Subtype

B

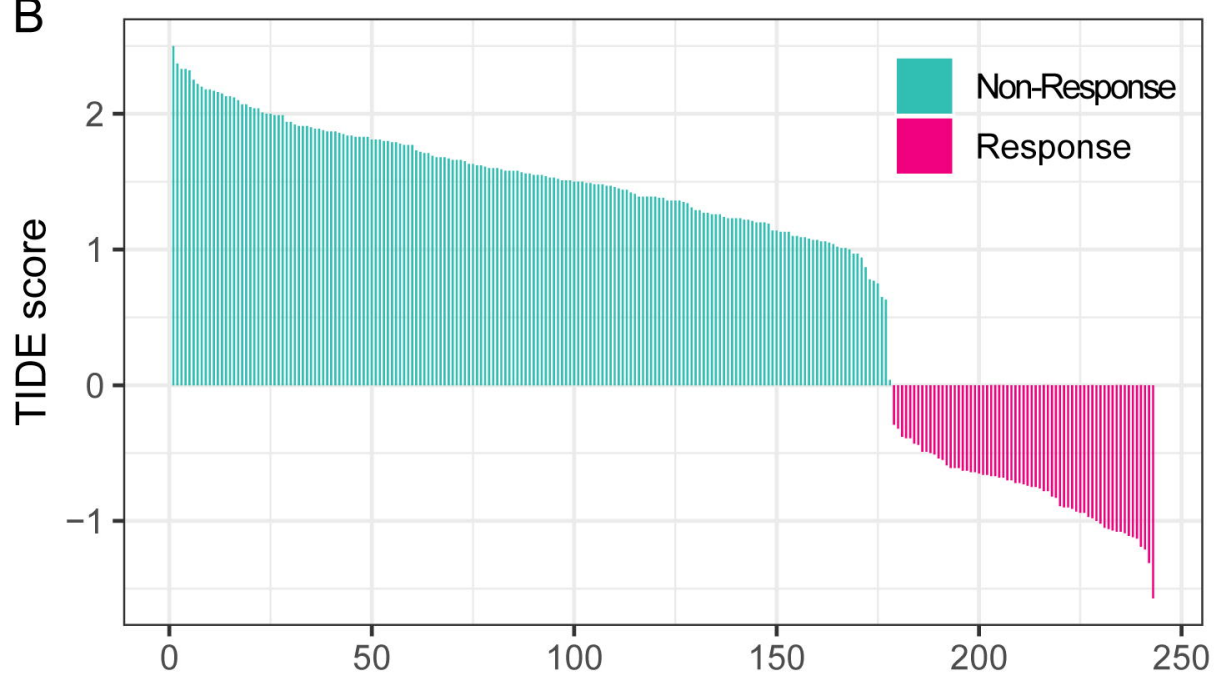

D
C
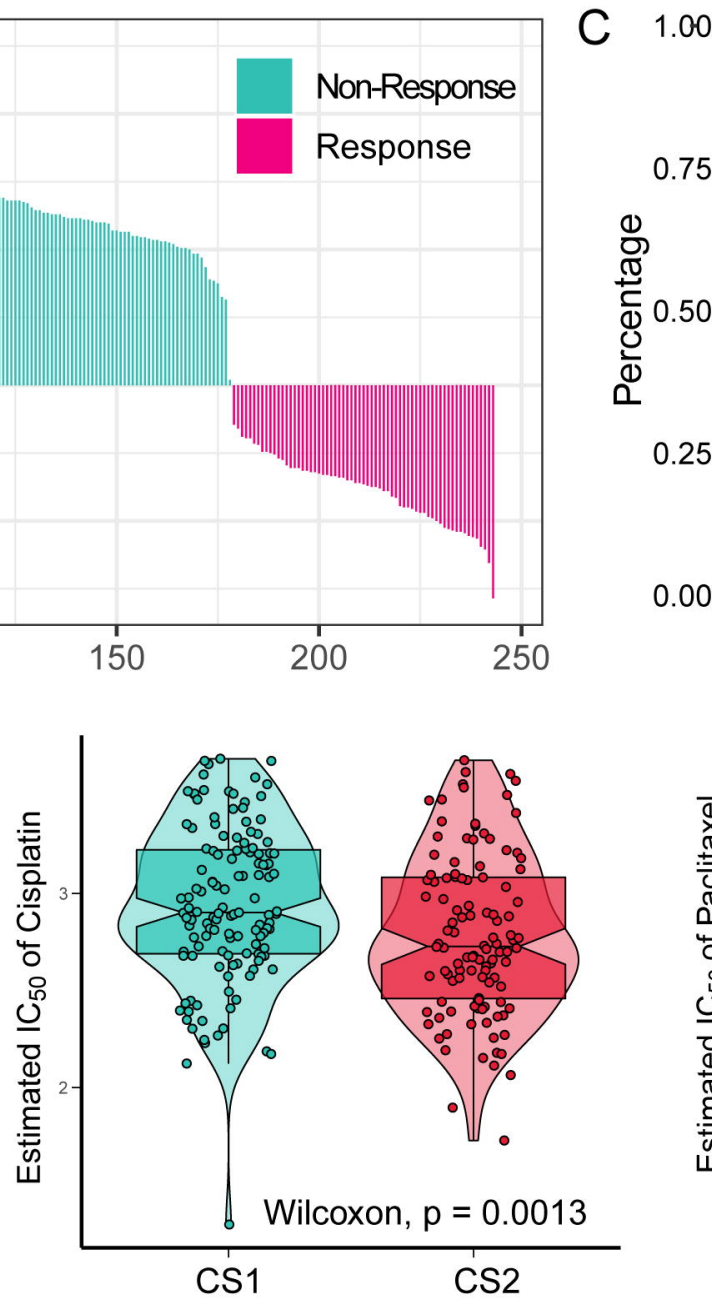

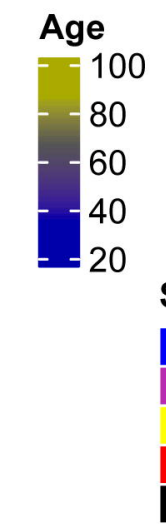

$\square$ unknown

0.5

0

$-0.5$

$-1$

Stage

Stage I

Stage II

Stage III

Stage IV
MALE

Grade

G1

G2

G3

GX

II
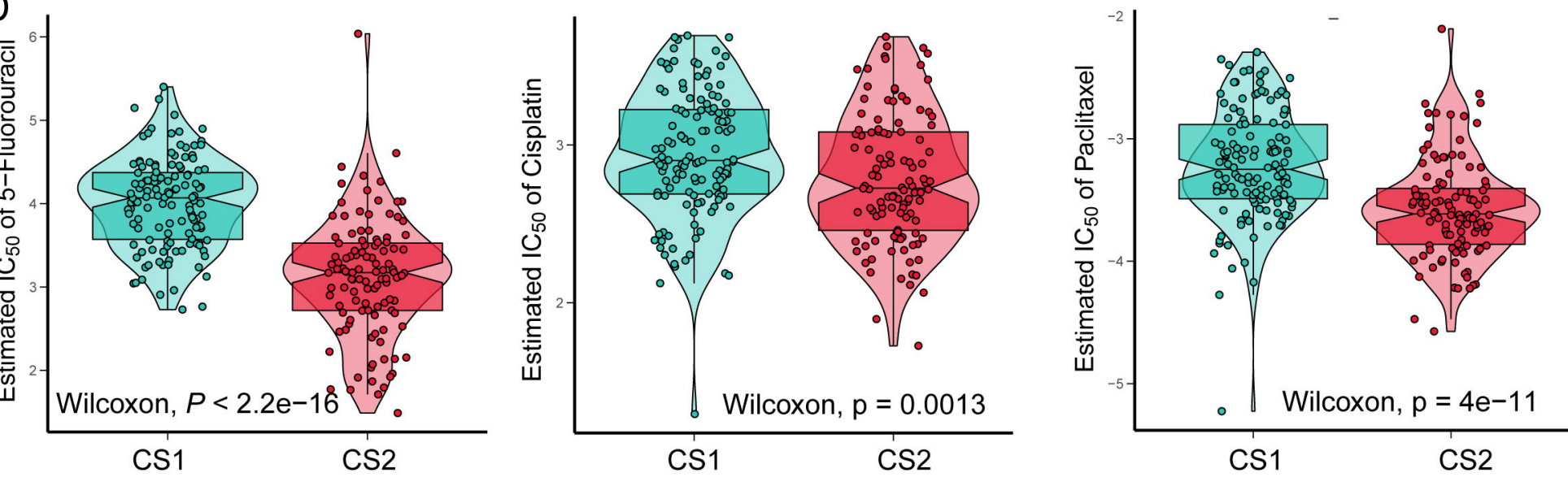

\section{Figure 6}


$A$
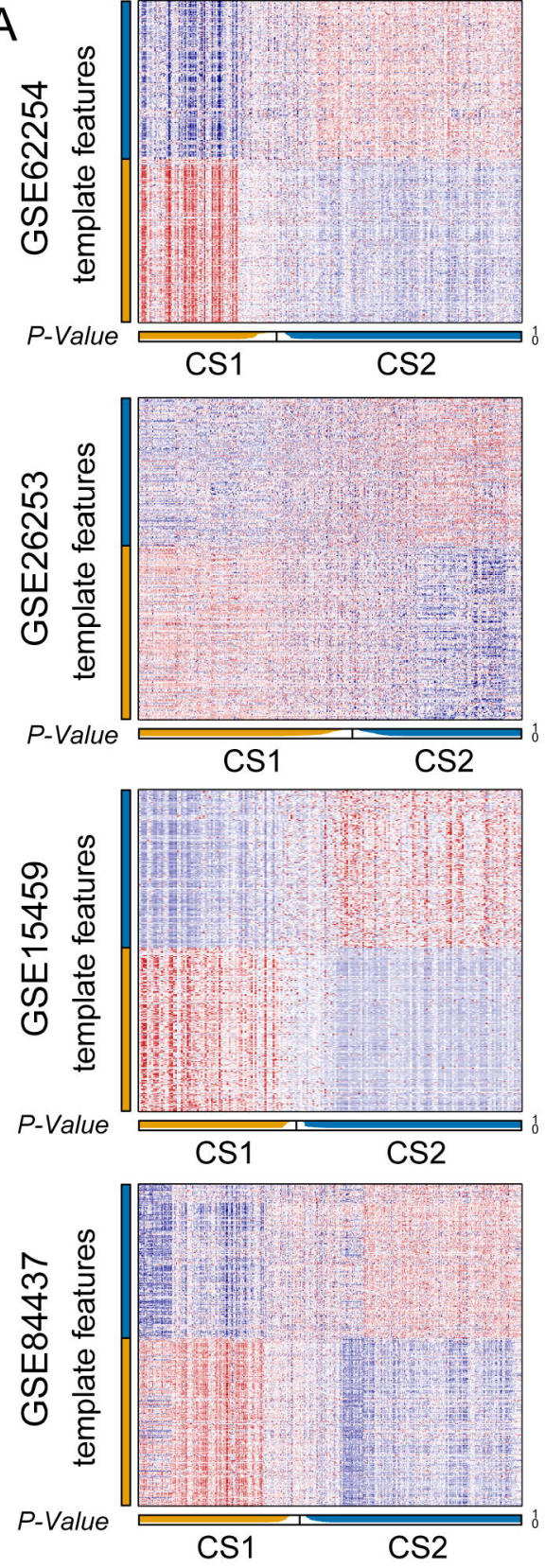

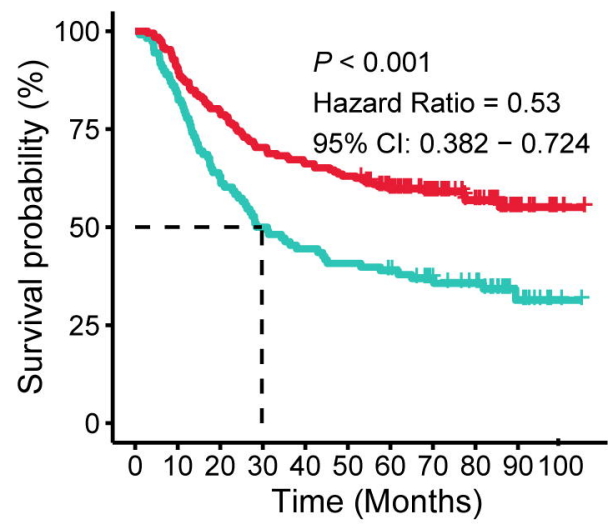

Number at risk

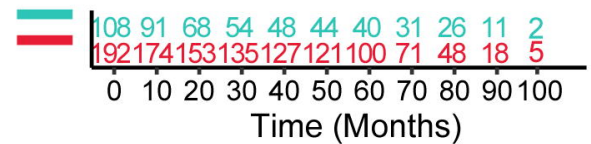
Time (Months)

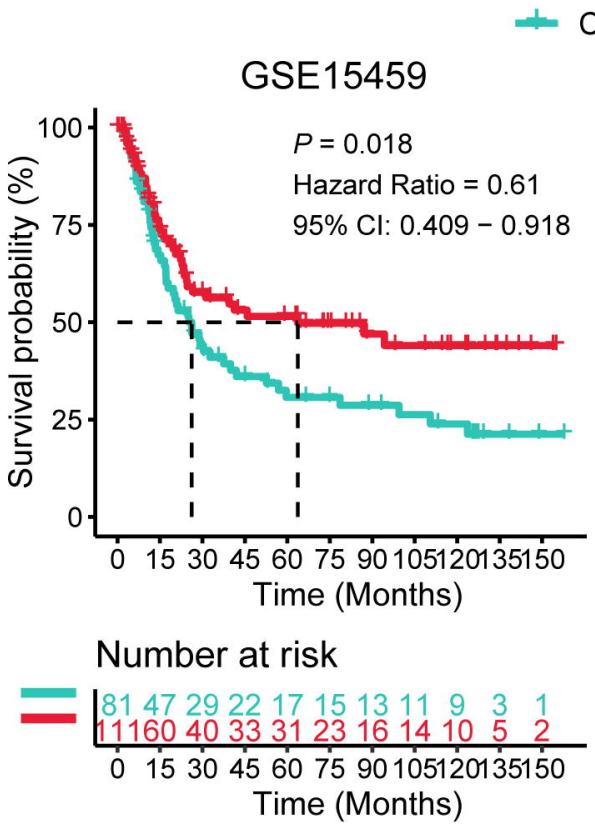

GSE26253

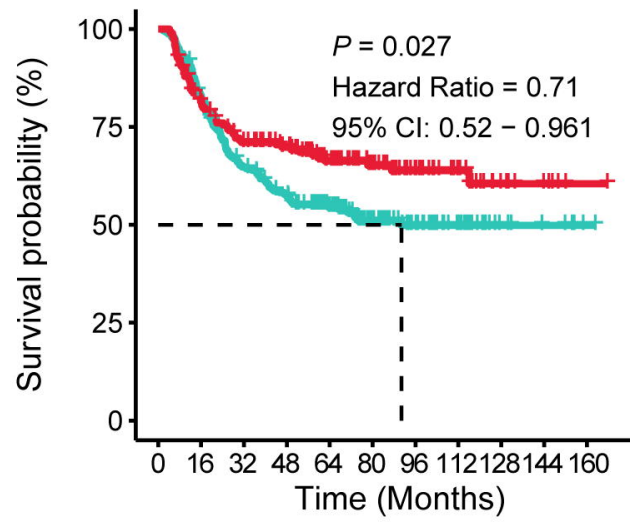

Number at risk

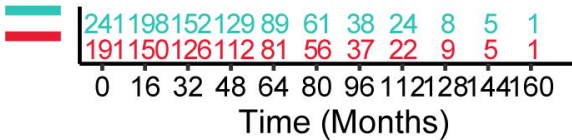

\section{GSE84437}

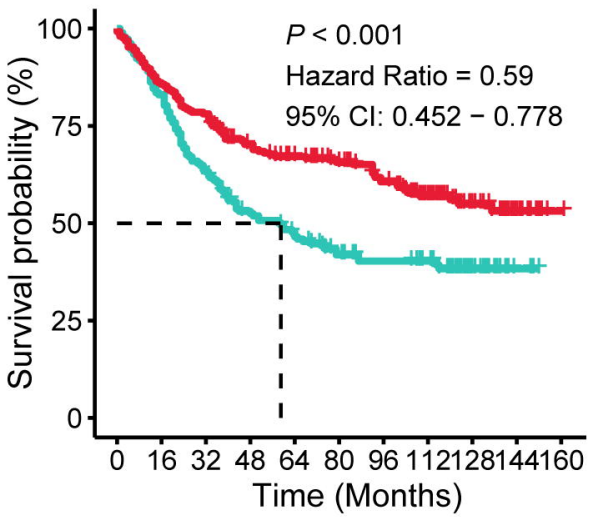

Number at risk

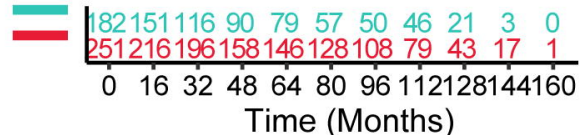

\section{Figure 7}

